\title{
Article
}

\section{Bacterial viability on chemically modified silicon nanowire arrays}

Susarrey-Arce, A., Sorzabal-Bellido, I., Oknianska, Alina, McBride, F., Beckett, A. J., Gardeniers, J. G. E., Raval, R., Tiggelaar, R. M. and Diaz Fernandez, Y. A.

Available at http://clok.uclan.ac.uk/33620/

Susarrey-Arce, A., Sorzabal-Bellido, I., Oknianska, Alina, McBride, F., Beckett, A. J., Gardeniers, J. G. E., Raval, R., Tiggelaar, R. M. and Diaz Fernandez, Y. A. (2016) Bacterial viability on chemically modified silicon nanowire arrays. Journal of Materials Chemistry B, 18 (4). pp. 3104-3112. ISSN 2050-750X

It is advisable to refer to the publisher's version if you intend to cite from the work. http://dx.doi.org/10.1039/C6TB00460A

For more information about UCLan's research in this area go to http://www.uclan.ac.uk/researchgroups/ and search for < name of research Group>.

For information about Research generally at UCLan please go to http://www.uclan.ac.uk/research/

All outputs in CLoK are protected by Intellectual Property Rights law, including Copyright law. Copyright, IPR and Moral Rights for the works on this site are retained by the individual authors and/or other copyright owners. Terms and conditions for use of this material are defined in the policies page.

\section{CLoK}

Central Lancashire online Knowledge www.clok.uclan.ac.uk

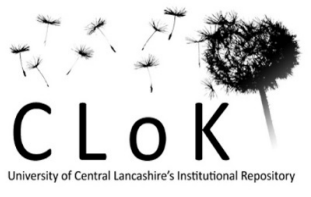




\section{Journal of \\ Materials Chemistry B}

Accepted Manuscript

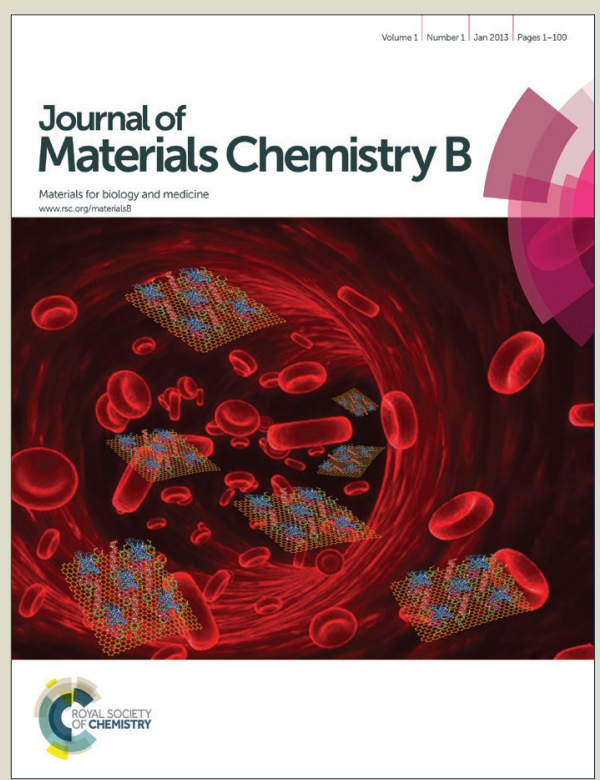

This is an Accepted Manuscript, which has been through the Royal Society of Chemistry peer review process and has been accepted for publication.

Accepted Manuscripts are published online shortly after acceptance, before technical editing, formatting and proof reading. Using this free service, authors can make their results available to the community, in citable form, before we publish the edited article. We will replace this Accepted Manuscript with the edited and formatted Advance Article as soon as it is available.

You can find more information about Accepted Manuscripts in the Information for Authors.

Please note that technical editing may introduce minor changes to the text and/or graphics, which may alter content. The journal's standard Terms \& Conditions and the Ethical guidelines still apply. In no event shall the Royal Society of Chemistry be held responsible for any errors or omissions in this Accepted Manuscript or any consequences arising from the use of any information it contains. 


\title{
Journal of Material Chemistry B
}

\section{ARTICLE}

\section{Bacterial viability on chemically modified silicon nanowire arrays}

Received 00th January 20xx, Accepted 00th January 20xx

DOI: $10.1039 / x 0 x \times 00000 x$

www.rsc.org/

\author{
A. Susarrey-Arce, ${ }^{* a}$ I. Sorzabal-Bellido, ${ }^{a}$ A. Oknianska, ${ }^{a}$ F. McBride, ${ }^{a}$ A. J. Beckett, ${ }^{b}$ J. G. E. \\ Gardeniers, ${ }^{c}$ R. Raval, ${ }^{* a}$ R. M. Tiggelaar, ${ }^{*}$, d Y. A. Diaz Fernandez, ${ }^{*}$
}

\begin{abstract}
The global threat of antimicrobial resistance is driving an urgent need for novel antimicrobial strategies. Functional surfaces are essential to prevent spreading of infection and reduce surface contamination. In this study we have fabricated and characterized multiscale-functional nanotopographies with three levels of functionalization: (1) nanostructure topography in the form of silicon nanowires, (2) covalent chemical modification with (3aminopropyl)triethoxysilane, and (3) incorporation of chlorhexidine digluconate. Cell viability assays were carried out on two model microorganisms $E$. coli and S. aureus over these nanotopographic surfaces. Using SEM we have identified two growth modes producing distinctive multicellular structures, i.e. in plane growth for $E$. coli and out of plane growth for $S$. aureus. We have also shown that these chemically modified SiNWs arrays are effective in reducing the number of planktonic and surface-attached microorganisms.
\end{abstract}

\section{Introduction}

Bacteria are one of the most abundant forms of life on our planet, ${ }^{1}$ and display a natural tendency to adhere onto surfaces as a selfdefence and proliferation mechanism. ${ }^{2}$ After initial colonisation of the surface, bacterial colonies undergo a change of metabolic activity that ultimately leads to the formation of complex multicellular structures, known as biofilms, which have a high level of defence against antimicrobial agents. ${ }^{3}$

Preventing bacterial adhesion on surfaces is a powerful strategy to control biofilm formation, ${ }^{4}$ reducing contamination of indwelling medical devices, ${ }^{5}$ assuring food security, ${ }^{6}$ and in developing antifouling coatings. ${ }^{7}$ The emergence of antimicrobial resistance (AMR) as a global threat has focused efforts in creating advanced materials and surfaces that can reduce and resist bacterial colonization. Promising antibacterial properties have been shown using chemical $^{8-10}$ or physical ${ }^{11-16}$ functionalities at surfaces. However, it is becoming increasingly clear that single approach strategies may be inadequate in combating bacterial attachment on surfaces, and there is an important need to create multifunctional systems combining two or more approaches. Here we develop a

\footnotetext{
a. Open Innovation Hub for Antimicrobial Surfaces at the Surface Science Research Centre and Department of Chemistry, University of Liverpool, Oxford Street, UK L69 3BX, Liverpool

b. Biomedical EM Unit, School of Biomedical Sciences, Crown Street, University of Liverpool, L69 3BX, Liverpool

c. Mesoscale Chemical Systems, MESA+ Institute for Nanotechnology, University of Twente, P.O. Box 217, 7500AE Enschede, The Netherlands

d. NanoLab Cleanroom, MESA+ Institute for Nanotechnology, University of Twente, P.O. Box 217, 7500AE Enschede, The Netherlands

*Corresponding authors: r.raval@liverpool.ac.uk, yuridiaz@liverpool.ac.uk, a.susarrev-arce@liverpool.ac.uk, r.m.tiggelaar@utwente.nl

Electronic Supplementary Information (ESI) available: [details of any supplementary information available should be included here]. See DOI: $10.1039 / \mathrm{x} 0 \times x \times 0000 \mathrm{x}$
}

material based on highly-oriented silicon nanowires (SiNWs) with three levels of functionalization, combining both nanotopographies and chemical functionalities. These hierarchical structures have demonstrated ability to work as scaffolds for living systems like mammalian cells, ${ }^{17-20}$ to aid bacterial-energy conversion ${ }^{21}$ and to facilitate molecular recognition. ${ }^{22}$ However, bacterial adhesion, biofilm formation and survival on SiNWs remain relatively underexplored. ${ }^{23-26}$ In this study we investigate the interaction and the viability of bacteria on SiNWs surfaces enhanced with different chemical functionalities, using model microorganisms $E$. coli and $S$. aureus.

\section{Materials and Methods}

\subsection{Fabrication of SiNWs}

SiNWs were fabricated on silicon substrates ( $p$-type boron doped, (100)-orientation, resistivity $5-10 \Omega \mathrm{cm}, 100 \mathrm{~mm}$ diameter, thickness $525 \mu \mathrm{m}$, single side polished; Okmetic, Finland). Prior to further processing, the substrates were cleaned by immersion in fuming 100\% nitric acid (UN2031; OM Group) for $10 \mathrm{~min}$. and in boiling 69\% nitric acid (BASF, 51153574) for 15min., which was followed by rinsing in demineralized water and spin drying. A regular pattern of $10 \mathrm{~mm} \times 10 \mathrm{~mm}$ samples was defined with UV-lithography, such that each sample comprised a centred area of $8 \mathrm{~mm} \times 8 \mathrm{~mm}$ in which SiNWs were formed. After development, the patterned resist was post-baked for at least $10 \mathrm{~min}$. at $120^{\circ} \mathrm{C}$ in ambient air. In the lithographically patterned areas SiNWs were formed with a twostep metal assisted chemical etching (MACE) process. ${ }^{27-30}$ A $50 \%$ aqueous HF (BASF-51151083) was diluted 5 times in in demineralized water (DI-water) to which $\mathrm{AgNO}_{3}$ (Sigma-Aldrich, 99\%) was added to a concentration of $0.005 \mathrm{M}$. In order to deposit $\mathrm{Ag}$ nanoparticles (AgNPs) on the exposed silicon areas, the patterned substrates are immersed (in the dark) in this solution for 
$1 \mathrm{~min}$. Subsequently the substrates are directly loaded in a solution of DI-water, 50\%-HF and $\mathrm{H}_{2} \mathrm{O}_{2}$ (BASF, 55316830) (volumetric ratio DI:HF: $\mathrm{H}_{2} \mathrm{O}_{2}=77.5: 20: 2.5$ ), and etched for 20min. (in dark conditions - etch rate ca. $0.6 \mu \mathrm{m} / \mathrm{min}$ ). After this, the substrates are rinsed with demineralized water. The AgNPs were removed by immersing the substrates in $69 \%$ nitric acid $\left(\mathrm{HNO}_{3} ; \mathrm{BASF}, 51153574\right)$ at room temperature for $65 \mathrm{~h}$, which was followed by rinsing with DI-water. To ensure complete removal of traces of photoresist the substrates were cleaned in a Piranha-solution (a 3:1 volumetric mixture of sulphuric acid $\left(\mathrm{H}_{2} \mathrm{SO}_{4} ; \mathrm{BASF}, \mathrm{UN} 1830\right)$ and $\mathrm{H}_{2} \mathrm{O}_{2}$; temperature $95^{\circ} \mathrm{C}$, cleaning time $15 \mathrm{~min}$.), after which they were rinsed in DI-water and dried with a nitrogen $\left(\mathrm{N}_{2}\right)$ flow. Finally individual samples of $10 \mathrm{~mm} \times 10 \mathrm{~mm}$ were cut using a dicing machine (Disco DAD-321), during which process glass plates covered the topside of the silicon substrates to avoid damage and contamination of the SiNWs. From SEM images (see Figure 1) the length, diameter and spacing between adjacent SiNWs were estimated for deeper layers with an error of 1- $\sigma$.

\subsection{Functionalization of SiNWs}

Chlorohexidine digluconate (CHD) and (3-aminopropyl) triethoxysilane (APTES) were purchased from Sigma Aldrich and used to introduce active chemical functionalities on the SiNWs surfaces. For the experiments described in sections 2.3 and 2.4, four kinds of SiNWs surfaces were prepared: (a) clean SiNWs, (b) SiNWs functionalised with CHD, (c) SiNWs functionalised with APTES, and (d) SiNWs functionalised with both APTES and CHD. The SiNWs surfaces with no additional functionalization were used after cleaning in $69 \%$ nitric acid. APTES functionality was introduced by immersing the SiNWs in $2 \%$ APTES in absolute ethanol for $2 \mathrm{~h}$ at $50^{\circ} \mathrm{C}$; followed by washing with ethanol and drying for $1 \mathrm{~h}$ at $50^{\circ} \mathrm{C}$. CHD functionalization was performed on clean SiNWs or SiNWsAPTES as required, by immersing the surfaces in aqueous solutions of $\mathrm{CHD}$ and allowing equilibration for $24 \mathrm{~h}$ at room temperature, followed by $15 \mathrm{~min}$ rinsing in DI-water, and air-drying at room temperature. For the experiments described in section $\mathbf{2 . 3}$ and $\mathbf{2 . 4}$, CHD loading concentration of $0.2 \%, 0.02 \%$, and $0.002 \%$ were used for the planktonic cells tests, whereas $0.02 \%$ and $0.002 \%$ were used to analyse the cells attached to the surface.

2.3 Determination of bacterial cell viability after culture of $E$. coli and $S$. aureus over SiNWs functionalised surfaces

S. aureus (DSM-346) and E. coli (ATCC-10798) cultures were grown overnight $\left(200 \mathrm{rpm}\right.$, at $37^{\circ} \mathrm{C}$ ) in NB medium (Oxoid, Ltd-Thermo Fisher). The bacterial cells concentration was adjusted to $10^{5}$ colony forming units per millilitre $(\mathrm{CFU} / \mathrm{mL})$ in sterile NB. Prior to microbiological assays, the surfaces investigated were immersed in $69 \% \mathrm{HNO}_{3}$ (Sigma-Aldrich) for $12 \mathrm{~h}$ and rinsed in $\mathrm{DI}$ water. $\mathrm{pH}$ was monitored to ensure complete $\mathrm{HNO}_{3}$ removal. Final $\mathrm{pH}$ values were found between 6.5 and 7.5. The surfaces were then placed in 24well cell culture plates and sterilized under UV light for $5 \mathrm{~min}$. Then, each substrate was immersed in $1 \mathrm{~mL}$ of bacterial cell suspension and incubated at $37^{\circ} \mathrm{C}$ for $0 \mathrm{~h}, 4 \mathrm{~h}, 8 \mathrm{~h}$ and $24 \mathrm{~h}$ for the determination of the viability of planktonic cells, and $8 \mathrm{~h}$ and $24 \mathrm{~h}$ for the viability experiments on surfaces. At the end of each incubation period, $\mathrm{CFU} / \mathrm{mL}$ colony counting of planktonic viable cells was performed
Thereafter, SiNWs surfaces were washed 3 times with sterile $0.85 \%$ $\mathrm{NaCl}$.

Cell viability of $S$. aureus and E. coli attached to SiNWs and to functionalised surfaces was analysed with Live/Dead BacLight Bacterial Viability kit (Molecular Probes, L7012). Briefly, all surfaces were stained for $30 \mathrm{~min}$ in the dark with $1 \mathrm{~mL}$ of sterile $0.85 \% \mathrm{NaCl}$ solution containing mixtures of SYTO 9 green-fluorescent nucleic acid stain for live cells, and the red-fluorescent nucleic acid stain propidium iodide for dead cells (see Scheme 1). After staining, surfaces were rinsed with sterile Milli- $Q$ water and immediately analysed using a confocal upright 880 multiphotone microscope. Collected images were analysed with a home-made script employing Fiji software.

\subsection{Scanning electron microscopy of bacterial cells attached to SiNWs functionalised surfaces}

Scanning electron microscopy (SEM) samples were initially processed as described in section 2.3, and fixed overnight at $4^{\circ} \mathrm{C}$ with $4 \%$ paraformaldehyde and $2.5 \%$ glutaraldehyde in $0.1 \mathrm{M}$ phosphate buffer. Subsequent fixation and staining were carried out in water using $2 \%$ osmium tetroxide for $1 \mathrm{~h}, 1 \%$ tannic acid for $30 \mathrm{~min}$., and $2 \%$ osmium tetroxide for $1 \mathrm{~h}$. Between each step, the samples were thoroughly rinsed with DI-water. Finally, staining overnight at $4^{\circ} \mathrm{C}$ with $1 \%$ uranyl acetate in water was performed. After staining, the samples were rinsed with DI-water, and progressively dehydrated with different volumetric ratios of ethanol (i.e. $30 \%, 50 \%, 70 \%, 90 \%$ and $100 \%$ ). After dehydration, SiNWs samples were critical-point dried in $\mathrm{CO}_{2}$ (Quorum Technologies K850) and sputter coated with $10 \mathrm{~nm}$ of AuPd (Quorum Technologies Q150T) for subsequent SEM imaging at $10 \mathrm{kV}$ with JEOL SEM 7001.

\section{Results and discussion}

Functional antibacterial surfaces can be classified into two main areas: (1) nanotopographic surfaces with intrinsic antibacterial properties, and (2) surfaces displaying chemical functionalities with biocidal effect. ${ }^{10,11,16}$ The combination of these two strategies may lead to a new generation of hierarchical multifunctional surfaces. To explore their potential, we have selected vertically aligned SiNWs as starting material with a defined topography and high surface area (see scheme 1(I)). We have added to these surfaces the chemical functionality APTES (see scheme 1(II)) which is frequently used in modification of biocompatible surfaces. ${ }^{31}$ Furthermore, exploiting the high surface area of our fabricated nanoarchitectures, we have used the SiNWs-APTES materials as carrier for the loading/release (see scheme 1(III)) of the biocide chlorohexidine digluconate (CHD). Bacterial viability of cells in the planktonic state and attached to surfaces was investigated and the individual effect of each functionality is described below.

\subsection{SiNWs nanotopographies}

Following our fabrication protocol, we are able to create nanotopographic structures on flat silicon wafers using a two-step metal assisted chemical etching process. ${ }^{27-30}$ These arrays of vertically aligned SiNWs are connected at the base with the silicon 
wafer, and can be obtained over a large area (i.e. $0.64 \mathrm{~cm}^{2}$ ). From SEM characterization in Figure 1, a thickness of the SiNWs-layer was estimated to be $11.3 \pm 0.2 \mu \mathrm{m}$. The top of each nanowire is observed to be slightly tapered, sharpening over a length of $c a .0 .7-1.1 \mu \mathrm{m}$. The diameter and spacing between adjacent SiNWs are $143 \pm 24 \mathrm{~nm}$ and $77 \pm 16 \mathrm{~nm}$, respectively. Top view SEM-images (Figure 1(b)) reveal that the SiNWs are slightly bent towards neighbouring wires. In fact, the tops of adjacent SiNWs clump together, forming a cauliflower-like pattern across the entire surface of the sample, which we refer to as 'regular crowding of SiNWs' (Figures 1(a) and (b)). The chemical composition of these surfaces was investigated

using XPS) and the results show that the surface is mainly composed of $\mathrm{SiO}_{2}$ (ca. 78\%, see ESI section 1). After overnight cleaning with nitric acid, the contact angle was $5^{\circ}$, indicating that the surface is superhydrophilic (ESI section 1). ${ }^{32}$

Comparable nanotopographies have been reported to have intrinsic antibacterial properties, attributed exclusively to morphological features. ${ }^{11}$ To evaluate the potential antibacterial properties of SiNWs, we have investigated the bacterial viability of two model microorganisms Escherichia coli (E. coli, Gram negative, rod-like shape) and Staphylococcus aureus (S. aureus, Gram positive, roundlike shape) $)^{33,34}$ on SiNWs arrays (scheme 1 ).
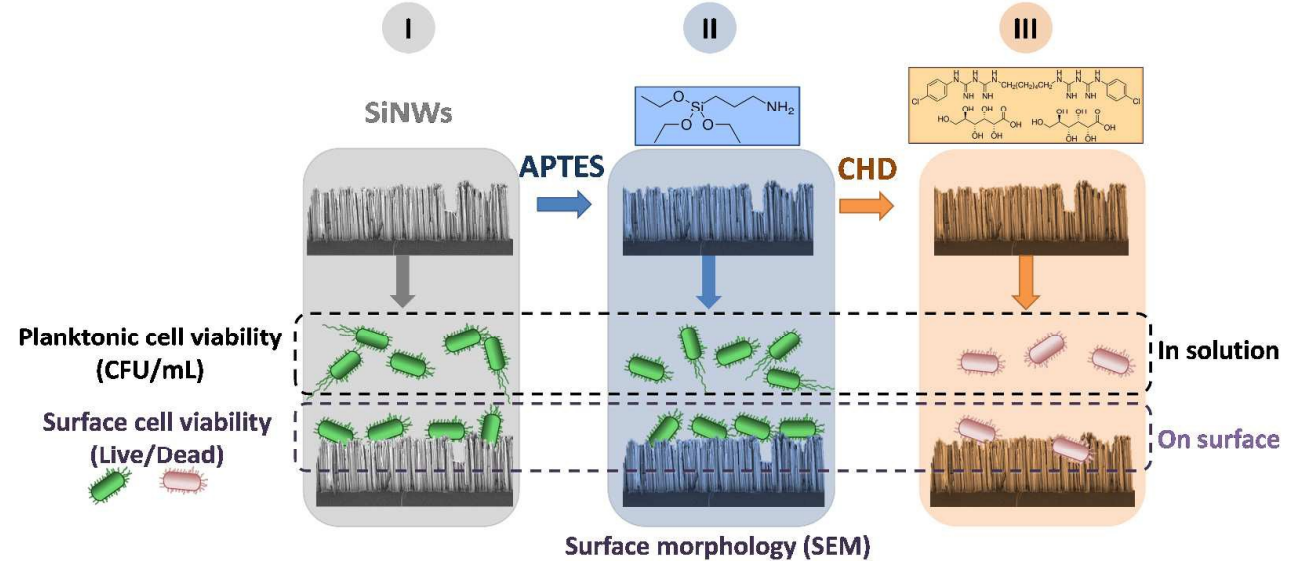

Scheme 1. Side-view SEM-images (false colour) of SiNWs (grey) functionalized with APTES (blue) and APTES loaded with CHD (orange). In the dashed open-black box: $\mathrm{CFU} / \mathrm{mL}$ colony counting of planktonic viable cells cultured over SiNWs samples. Analysis of bacterial cell viability on surfaces (purple dashed box) was performed by Live/Dead staining followed by confocal microscopy. The morphology of the cells attached to surfaces was examined by SEM.
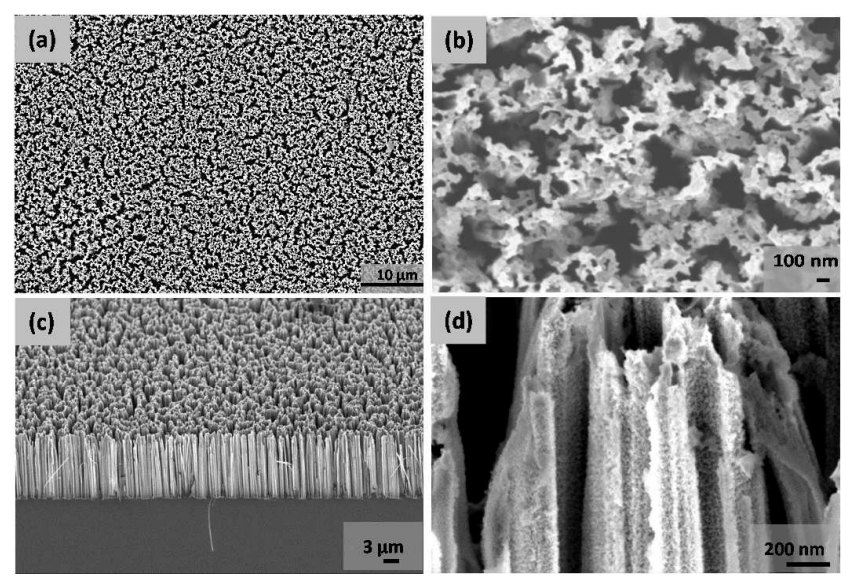

Figure 1. Representative SEM images of: (a-b) Top-view of SiNWs. (c-d) Side-view of SiNWs.

\subsubsection{Bacterial colonisation on SiNWs}

Viability of cells attached to SiNWs arrays was investigated using Live/Dead staining as sketched in scheme $\mathbf{1}(\mathbf{I})$. Confocal microscopy images in Figure $\mathbf{2}$ show that the majority of E. coli and S. aureus deposited cells remained alive (green) after $8 \mathrm{~h}$ of culture over SiNWs, while a smaller amount of cells were identified as dead (red) as shown in Figures 2.

To generate additional insights on bacterial interaction with the surfaces, we have performed SEM analysis. Figure $\mathbf{3}$ displays a sideview SEM image of $E$. coli on SiNWs, where intact, morphologically unchanged cells lay horizontally over the SiNWs surface. These results are in agreement with the viability data as shown in Figure 2 demonstrating that nanofabricated surfaces display relatively low, but measurable, antibacterial activity. Surface nanotopography has been proven to display intrinsic antibacterial properties as reported by Ivanova et al. on black silicon surfaces, ${ }^{11}$ where compromised bacterial cell membranes were observed, attributed to the formation of toroidal pores in a lipid bilayer. 

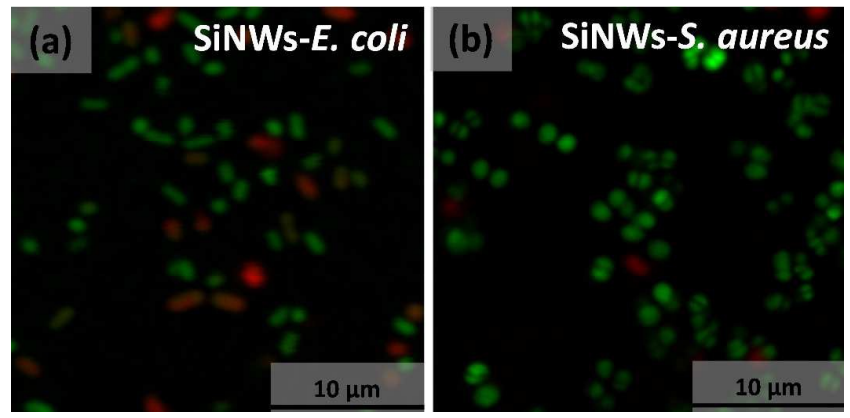

Figure 2. Representative merged confocal laser scanning microscope images for live (green), and dead (red) bacteria on SiNWs after $8 \mathrm{~h}$ of culture: E. coli (a) \& S. aureus (b).

Our non-functionalised SiNWs arrays demonstrate lower antibacterial activity with respect to black silicon. This may be attributed to the differences in topography (e.g. structure height, diameter, and interspacing between structures) which represent a key factor in the surface performance. ${ }^{15}$ SEM images of our SiNWs surfaces show relatively high wires density, with average wire-wire interspacing of $77 \mathrm{~nm}$. Under these conditions, both E. coli and $S$. aureus attach on top of the SiNWs arrays. Chemical composition could play also an important role on the antibacterial performance of these surfaces and our SiNWs arrays contain up to $78 \%$ of $\mathrm{SiO}_{2}$ (see ESI data). These differences suggest that both topography and surface chemistry should be considered for the development of new nanostructured surfaces with antibacterial properties.

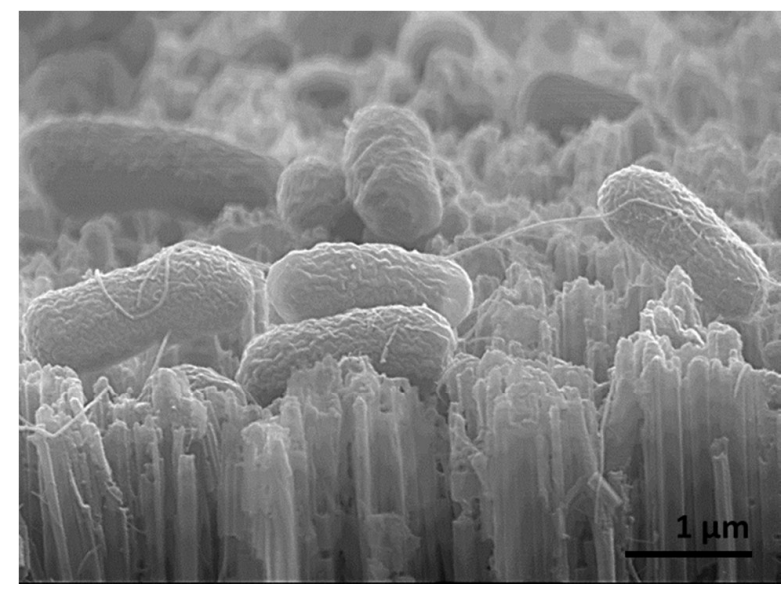

Figure 3. Representative side-view SEM image of $E$. coli on culture for 8h over SiNWs.

\subsection{Bacterial attachment on SiNWs-APTES surfaces}

Functionalization of silicon oxide surfaces with silanes for biological applications is well known and has been previously discussed in the literature. $^{35}$ APTES is a small molecule that can be used to covalently bind biologically active molecules to $\mathrm{SiO}_{2}$ surfaces. Here, we functionalized SiNWs surfaces with APTES to incorporate chemical functionalities to our nanotopographies as shown in Scheme 1(II).

Live/Dead viability assays on cells attached to the surface of SiNWsAPTES (Figure 4) show similar results to experiments obtained with non-functionalized SiNWs. Bacteria are able to survive and colonise the surface, irrespective of APTES functionalization. These results suggest that the SiNWs arrays modified with APTES also have a relatively low intrinsic antibacterial activity, and that the presence of APTES does not significantly alter the bacterial viability.of the SiNWs arrays..
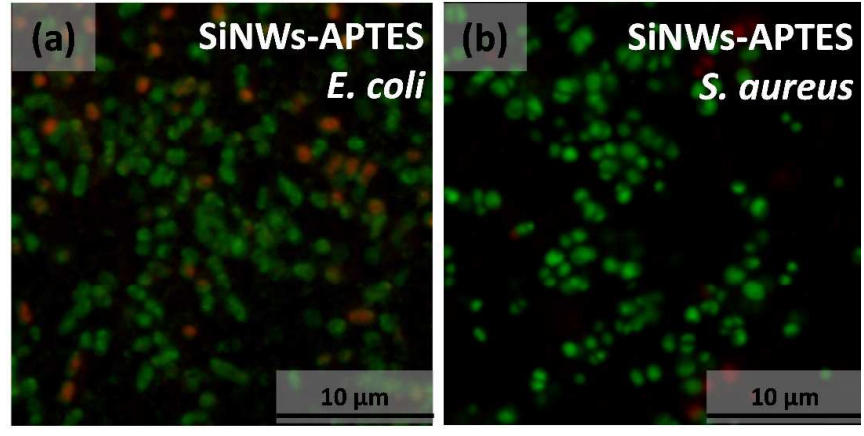

Figure 4. Representative merged confocal laser scanning microscope images of live (green) and dead (red) bacteria on SiNWs-APTES after 8 h of culture: (a) E. coli \& (b) S. aureus.

\subsubsection{Growth modes of $E$. coli on SiNWs and SiNWs-APTES}

SEM characterisation of bacteria attachment to SiNWs and SiNWsAPTES surfaces was also carried out. The sample preparation protocol preserves the morphology of the attached cells and extracellular matrix components involved in attachment with a high level of detail. High magnification SEM images on E. coli samples (Figure 3) show that the rod-like cells lie horizontally on the SiNWs arrays, without compromising cell morphology. The results from SiNWs-APTES, Figure 5, were also similar, suggesting that the APTES functionality does not alter cell behaviour. Direct interaction of cells with the surface appears to be favoured in both cases, producing in plane 'two-dimensional (2D)' bacterial colonies. From SEM images, we do not observe dramatic cell damage induced by interaction with the sharp features of SiNWs arrays (Figure $\mathbf{3}$ and $\mathbf{5}$ ).

The morphology of the colonies may be associated with both the interaction of $E$ Coli with the surface and the growth mode of $E$. coli. For Gram negative E. coli many adhesins are displayed on pili or fimbriae, which are hair-like appendages on bacterial cells that allow single bacterial cells to attach to surfaces. The SEM image in Figure 3 shows that $E$. coli cells take advantage of flagella and pili to attach to sharp tips and cavities to colonise the surface..$^{36-38}$ These extracellular structures and the rod-like shape of $E$. coli cells can generate multiple contact points with the irregular SiNWs arrays, as observed in our SEM data and as reported on other surfaces ${ }^{39}$. 


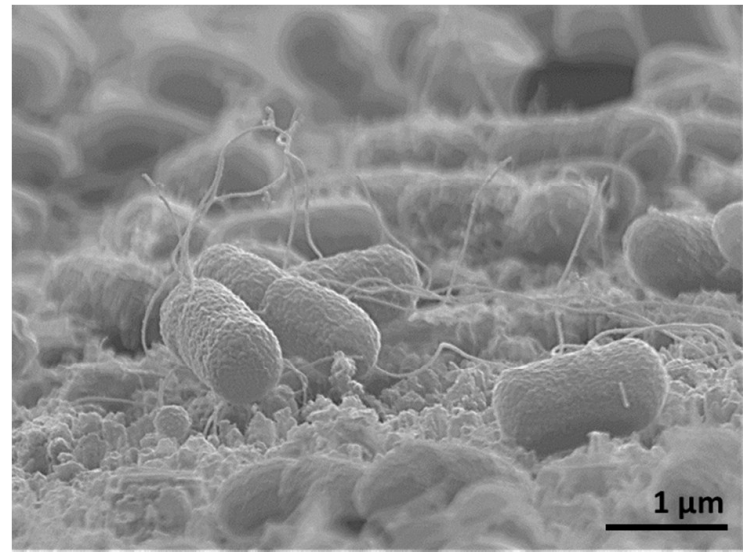

Figure 5. Representative side-view SEM image of E. coli on SiNWs functionalized with APTES after 8h of culture.

We hypothesize that after initial attachment, E. coli cells predominantly align and orientate parallel to the surface plane due to multiple bacteria-surface anchoring points as shown in scheme 2(a) and SEM images in Figures 3 \& 5, and ESI Figure S3.
Furthermore, E coli cells display a symmetric rod-like shape ${ }^{36,37}$ with a single polar axis and are known to undergo lateral growth following the direction of the polar axis. ${ }^{37,} 38$ Therefore, preferential orientation of $E$. coli parallel to the surface plane would directly lead to '2D structures'. Interestingly, $E$. coli cells on flat silicon surfaces (ESI Figure S3) also display similar morphological structures, suggesting that these bacteria can accommodate to a range of surfaces, with the rods preferentially aligning along the surface plane. Live-dead assays on the flat Si surfaces, however, show no kill, suggesting that the small bactericidal activity observed in the SiNW and APTES-functionalised SiNW arrays can be attributed to the nanotopography.

\subsubsection{Growth modes of $\mathrm{S}$.aureus on SNWs and SiNWs-APTES}

In contrast to E. coli cells, S. aureus colonies on SiNWs and SiNWsAPTES surfaces develop vertically, displaying only a few contact points with the surface, and showing preferential out-of-the-plane growth (Figure 6, scheme 2(b)), resulting in three dimensional (3D) morphologies.. The APTES functionalization, again, does not appear to affect the behaviour at the nanotopographic arrays.
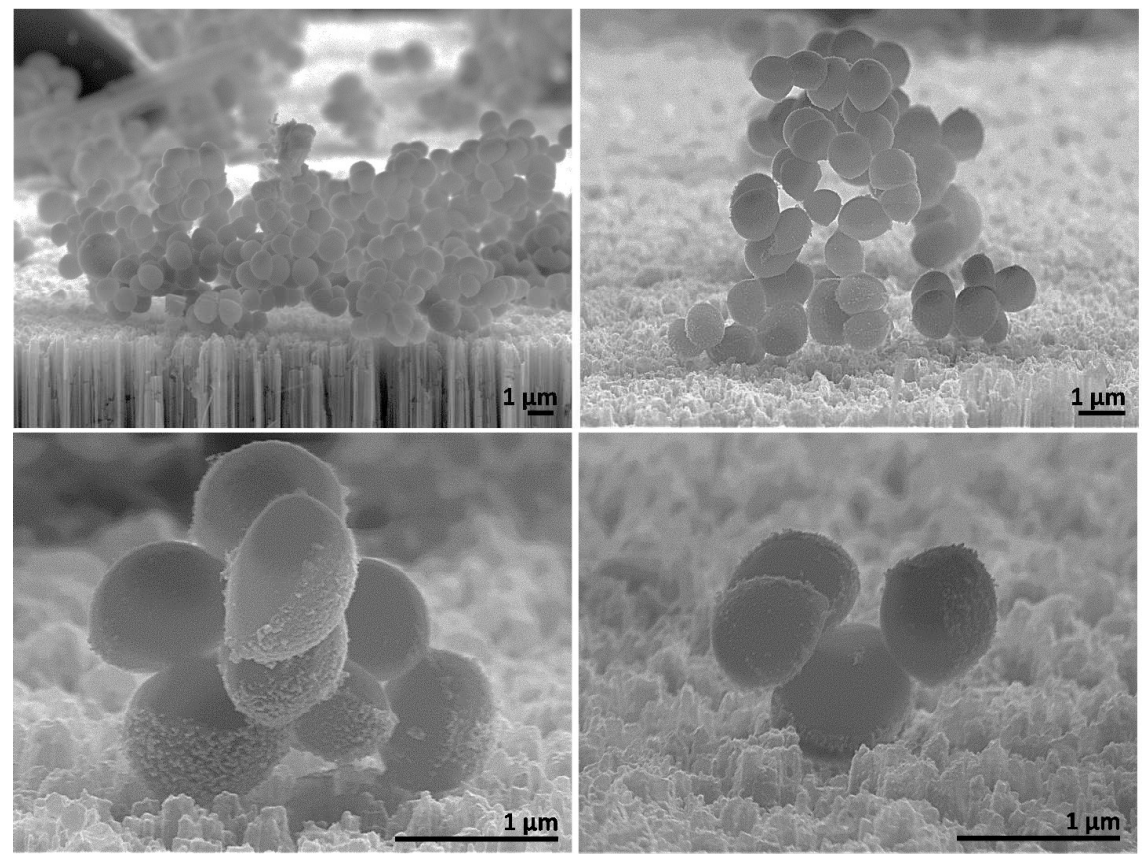

Figure 6. Representative side-view SEM image of $S$. aureus on SiNWs-APTES after $8 \mathrm{~h}$ of culture. Interestingly, even very small colonies and delicate three-dimensional structures are able to resist sample processing for SEM, suggesting that cell/cell and cell/surface interactions are relatively strong. ESI Figure $\mathbf{S 4}$ shows similar morphologies for $S$. aureus on the SiNWs without APTES.

S. aureus attachment on abiotic surfaces can be mediated by adhesins covalently anchored to the cell wall, allowing both cellcell, and cell-surface attachment. The round shape of the cell enables only small contact areas to form with irregular surfaces like SiNWs arrays (Figure 6, Figures S4). Furthermore, S. aureus displays a cell membrane composed of a highly rigid peptidoglycan layer ${ }^{38-39}$, which prevents the cells from adapting readily to surface topographies, which would affect cell-surface attachment and also the capability of bacterial growth along the surface plane. The SEM data of Figure 6 suggests that there are relatively few cell-tosurface attachments, with out-of-plane growth predominating and leading to vertical multi-cellular colonies on SiNWs arrays, as shown 
in Scheme 2(b). In contrast, on flat silicon, S. aureus displays more cell-surface attachments (Figure S5), suggesting that surface topography influences the attachment behaviour of this microorganism.

\section{(a) E. coli}

In plane growth

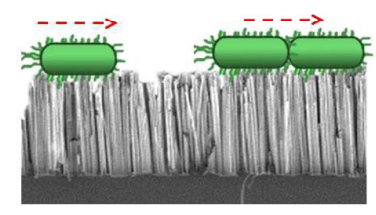

(b) S. aureus

\section{Out of plane growth}

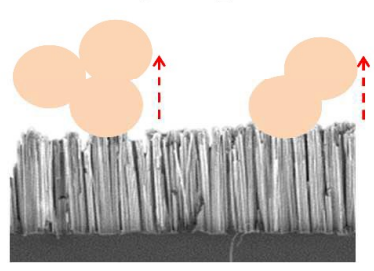

Scheme 2. Representation of bacterial growing modes on SiNWs \& SiNWs-APTES surfaces: (a) E. coli and (b) S. aureus

3.3 SiNWs surfaces for biocidal release: cell viability on SiNWs and SiNWs-APTES arrays functionalised with CHD

The high surface area of SiNWs arrays shown in Figure 1, makes them good candidates for carrying bioactive cargos. ${ }^{18-20}$ We have exploited this property to add a third level of functionalization to SiNWs surfaces by using chlorhexidine digluconate (CHD), which is widely employed as a surface disinfectant and topical antibacterial agent. ${ }^{40-41}$ Since CHD can be released from the surface, it has the potential to affect the viability of cells directly attached to the surface, as well as planktonic cells dispersed in the liquid medium. Therefore, in the next sections, we have studied the effect of CHD functionalization by evaluating the antibacterial activity against $E$. coli and $S$. aureus in the planktonic state. Subsequently, the cell viability of surface attached E. coli and S. aureus was evaluated using live/dead staining, according to the strategy described in Scheme 1(III).

\subsubsection{Planktonic cell viability on SiNWs and SiNWs-APTES arrays} loaded with CHD

We have considered two key factors that influence the antimicrobial properties of our surfaces: a) the loading capacity of the porous surface, and b) the released concentration of CHD over time into the liquid medium. Generally, the amount of material at the surface is negligible with respect to the liquid environment. Therefore, low loading capacity can lead to depletion of the cargo before reaching a critical microbicidal concentration, while excessively high loading causes unnecessary release of biocide to the environment, risking the emergence of AMR. On the other hand, a relatively fast release of the cargo could deactivate the surface, allowing recolonization; while for relatively slow release, the biocide concentration over time might not be sufficient to exhibit antibacterial activity. Therefore, an optimal balance between cargo loading and release rate is necessary to deliver optimal performance of the antimicrobial surfaces.

In order to evaluate the effects of these factors on the released concentrations of CHD from SiNWs and SiNWs-APTES surfaces, we have performed a multi-level assay by varying the initial loading concentration of CHD from $0.002 \%$ to $0.2 \%$, and collecting kinetic values of $\mathrm{CHD}$ release into the bulk medium after $4 \mathrm{~h}, 8 \mathrm{~h}$, and $24 \mathrm{~h}$. The released concentrations were evaluated using UV-Vis spectroscopy, exploiting the intense absorption peak of CHD at $255 \mathrm{~nm}$ (ESI Figure S6), using the same volume/surface ratio employed in the bioassays (see experimental section for details). The CHD quantification limit for this method is estimated as $\sim 0.4 \mathrm{mg} / \mathrm{L}$. We observed no difference in the release from $4 \mathrm{~h}$ to $24 \mathrm{~h}$, suggesting that the CHD release is completed within $4 \mathrm{~h}$. The final concentration of CHD released from SiNWs-APTES is higher than from SiNWs surfaces, see Table 1. In addition to these results, we have also determined the minimum inhibitory concentrations (MIC) of CHD for the bacterial strains, obtaining values of $0.3 \mathrm{mg} / \mathrm{L}$ for $E$. coli and $0.6 \mathrm{mg} / \mathrm{L}$ for $S$. aureus. Considering the release data from Table 1, we can conclude that the concentration of $\mathrm{CHD}$ released into the liquid medium is above the $E$. coli MIC value for both SiNWs and SiNWs-APTES samples using loading concentrations of $0.2 \%$, whereas for loading $0.02 \%$, only SiNWs-APTES exceeded the MIC values for E. coli. For S. aureus, only SiNWs-APTES loaded with $0.2 \%$ of $\mathrm{CHD}$ exceeded the MIC value, while $0.02 \%$ loading produced a released concentration nominally close to the MIC.

Table 1. Release of $\mathrm{CHD}$ from surfaces at different loading concentrations after $4 \mathrm{~h}$ in $\mathrm{H}_{2} \mathrm{O}$

\begin{tabular}{|l|l|l|}
\hline Surface & SiNWs & SiNWs-APTES \\
\hline CHD Loading & Release & Release \\
$0.2 \%$ & $0.52 \mathrm{mg} / \mathrm{L}$ & $2.0 \mathrm{mg} / \mathrm{L}$ \\
$0.02 \%$ & $<0.4 \mathrm{mg} / \mathrm{L}^{\S}$ & $0.6 \mathrm{mg} / \mathrm{L}$ \\
$0.002 \%{ }^{\S}$ & $<0.4 \mathrm{mg} / \mathrm{L}^{\S}$ & $<0.4 \mathrm{mg} / \mathrm{L}^{\S}$ \\
\hline${ }^{5}$ below the detection limit of CHD & \\
\hline
\end{tabular}

To assess antimicrobial activity of the released CHD in the planktonic state, we determined the number of colony forming units (CFU/mL) for bacterial solutions cultured for $4 \mathrm{~h}, 8 \mathrm{~h}$, and $24 \mathrm{~h}$ over SiNWs and SiNWs-APTES loaded with various concentrations of CHD (see Figure 7). Internal control samples for Oh incubation were included in each set of experimental data (red bars in Figures 7a-d). The results presented in Figure 7 demonstrate that in the absence of $\mathrm{CHD}$, no significant difference in planktonic bacterial viability was observed between SiNWs and SiNWs-APTES surfaces for both $E$. coli and $S$. aureus, suggesting that, under our experimental conditions, the functionalization of SiNW with APTES only does not have any effect on the planktonic cells.

When the SiNWs-APTES arrays are functionalised with CHD, antibacterial effects are observed and the different sensitivity of $E$. coli and $S$ aureus is manifested. For loading concentrations of $0.2 \%$ of $\mathrm{CHD}$, both S. aureus and E. coli are completely killed (Figure 7 WACO), as expected from the concentration of $\mathrm{CHD}$ released from these surfaces $(2.0 \mathrm{mg} / \mathrm{L})$, which is considerable higher than the MIC value of both microorganisms. Lower CHD loadings eradicate planktonic E. coli on SiNWs-APTES-CHD, but failed to completely kill S. aureus in the planktonic state (Figure 7b SiNW-AC1 \& SiNW-AC2). Interestingly, for these low loading concentrations, S. aureus 
showed in Figure 7 an initial biocidal effect from $0 \mathrm{~h}$ to $8 \mathrm{~h}$, followed by an increase in the cell population by four orders of magnitude from $8 \mathrm{~h}$ to $24 \mathrm{~h}$. These results can be explained considering the antibacterial mechanism of action of CHD. Cheung et al. reported that $\mathrm{CHD}$ biguanide kills Gram positive and negative microorganism by affecting cell membrane permeability. These authors have also demonstrated that the killing effect increases at longer biocide exposure time leading to progressive deterioration of cell membrane. ${ }^{41}$ We hypothesise here similar effects for chlorhexidine digluconate to explain the progressive reduction of bacterial population from $0 \mathrm{~h}$ to $8 \mathrm{~h}$ (Figure $\mathbf{7}(\mathbf{b})$ ). The cell regrowth observed from $8 \mathrm{~h}$ to $24 \mathrm{~h}$ can be attributed to the presence of live bacteria either attached to the surface or surviving in liquid medium. This explanation is consistent with the concentrations of $\mathrm{CHD}$ released from the surfaces for $0.02 \%$ or lower loading in Table 1, leading to concentrations of CHD in the growing medium close to or below the MIC value for $S$. aureus.

The planktonic viability assay also demonstrates that the functionalization of SiNWs with APTES increases the antibacterial activity of the surfaces against planktonic cells, as expected from the higher concentration of CHD released from SiNWs-APTES surfaces (Table 1). These results are also consistent with SEM data, presented in the following section, showing lower density of bacterial cells attached to the surface of SiNWs-APTES-CHD (Figure 8) with respect to SiNWs-CHD (Figure 9) for the same CHD loading concentration.
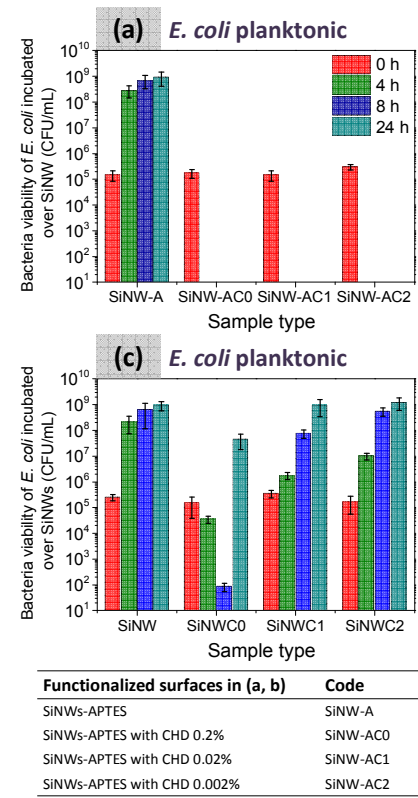

Figure 7. Planktonic viability for $E$. coli and S. aureus after different culture times. Sample acronyms are listed within the table.

(d) S. aureus planktonic

\subsubsection{Bacterial viability on SiNWs \& SiNWs-APTES arrays} functionalised with CHD

From the planktonic viability data presented in section 3.3.1, we have identified SiNWs-APTES functionalised with CHD as our most promising candidate antibacterial surface. For this reason we have evaluated bacterial attachment on SiNWs-APTES and SiNWs-APTESCHD using SEM imaging after different bacteria culture times. For each culture time SiNWs or SiNWs-APTES was used as a control. The results in Figure $\mathbf{8}(\mathbf{a}, \mathbf{c}, \mathbf{e})$ show representative SEM images of $E$. coli and S. aureus cell attachment to the SiNWs-APTES arrays after 8 and $24 \mathrm{~h}$. The extent of surface attachment increases with the culture time, displaying low bacterial coverage up to $4 \mathrm{~h}$ (data not shown), while for longer culture times, dense bacterial colonies were observed along the surface.

On SiNWs-APTES-CHD arrays loaded with $0.02 \%$ of CHD, SEM analysis demonstrates that for both $E$. coli and $S$. aureus, the number of cells attached to the surface after $8 \mathrm{~h}$ is negligible compared to the SiNWs-APTES control (Figures $\mathbf{8} \mathbf{a}, \mathbf{b}$ ). For E. coli, the surface-based antibacterial activity was maintained up to $24 \mathrm{~h}$ (ESI-Figure S8), while for S. aureus, we observe recolonization of the SiNWs-APTES-CHD arrays at 24 hrs (Figure 8f). These results echo the planktonic viability data in that the SiNW-APTES-CHD combination is also effective for delivering antibacterial effects at the surface.
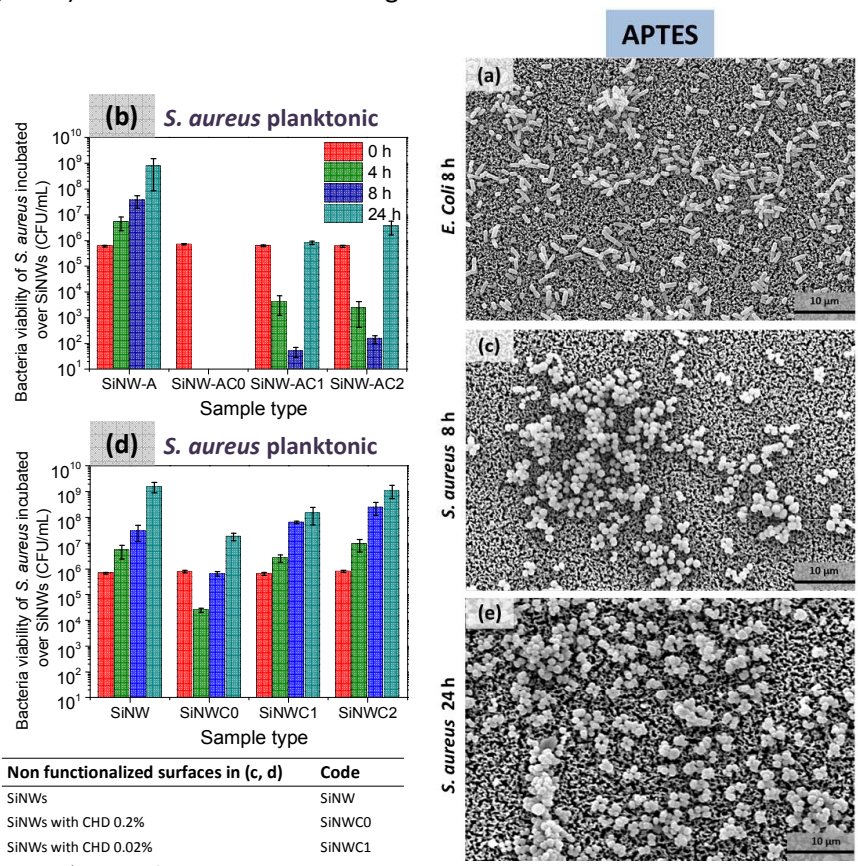

\begin{tabular}{ll}
\hline Non functionalized surfaces in (c, d) & Code \\
\hline SiNWs & SiNW \\
SiNWs with CHD $0.2 \%$ & SiNWCO \\
SiNWs with CHD 0.02\% & SiNWC1 \\
SiNWs with CHD 0.002\% & SiNWC2 \\
\hline
\end{tabular}

Figure 8. Representative top-view SEM image of $E$. coli after $8 \mathrm{~h}$ of culture on (a) SiNWs-APTES, (b) SiNWs-APTES-CHD loaded with $0.02 \%$ CHD; S. aureus (c-f) on SiNWs-APTES after (c) $8 \mathrm{~h}$ and (e) $24 \mathrm{~h}$ of incubation. S. aureus on SiNWs-APTES-CHD loaded with $0.02 \%$ of CHD after (d) $8 \mathrm{~h}$ and (f) $24 \mathrm{~h}$ of culture. 
From our data, it is clear that the presence of CHD is important for imparting antibacterial properties to our SiNWs and SiNWs-APTES arrays. However, the role of APTES functionalization, while not delivering an intrinsically antibacterial effect to the SiNWs surfaces, plays an important role in the antibacterial effect of CHD. To clarify this, we also investigated bacterial surface attachment on SiNWsCHD (Figure 9c-d), i.e. SiNWs arrays that are not functionalised by APTES. Although, there is a reduction of the number of cells attached compared to the SiNWs arrays, the performance of SiNWs-CHD is poorer when compared with the SiNWs-APTES-CHD counterparts (Figure 8). This suggests that APTES functionalization also plays an important role, when combined with CHD.

Following the strategy described in Scheme 1 (III), we have quantified the biocidal effect on cells attached to SiNWs and SiNWsAPTES-CHD, to generate further insights on their antimicrobial activity. Viability of cells attached to the surface was determined by live/dead staining (Figures 10, ESI Figures S9 \& S10). These results demonstrate that on SiNWs-APTES-CHD with $0.02 \% \mathrm{CHD}$ loading the fraction of live $S$. aureus cells remains below $5 \%$, despite the recolonization observed by SEM at $24 \mathrm{~h}$ (Figure $8 \mathrm{f}$ ). Even at lower CHD loading, the number of live cells is below 20\% (Figure 10b, SiNW-AC2), suggesting that the viability of attached cells is strongly compromised on SiNWs-APTES-CHD surfaces. For E. coli, Figure 10a shows that the percent of viable cells on SiNWs-APTES-CHD surfaces is systematically lower than for $S$. aureus, as expected from the higher sensitivity of $E$. coli to CHD. Similarly, for both bacteria, APTES functionalization increases the antibacterial activity against attached cells. It is also interesting to note that the intrinsic topological antibacterial effects of non-functionalised SiNWs and SiNWs-APTES account for 30\%-60\% reduction of bacterial viability, versus $10 \%-20 \%$ of dead cells on flat silicon (Si) and flat-siliconAPTES surfaces (Si-A).

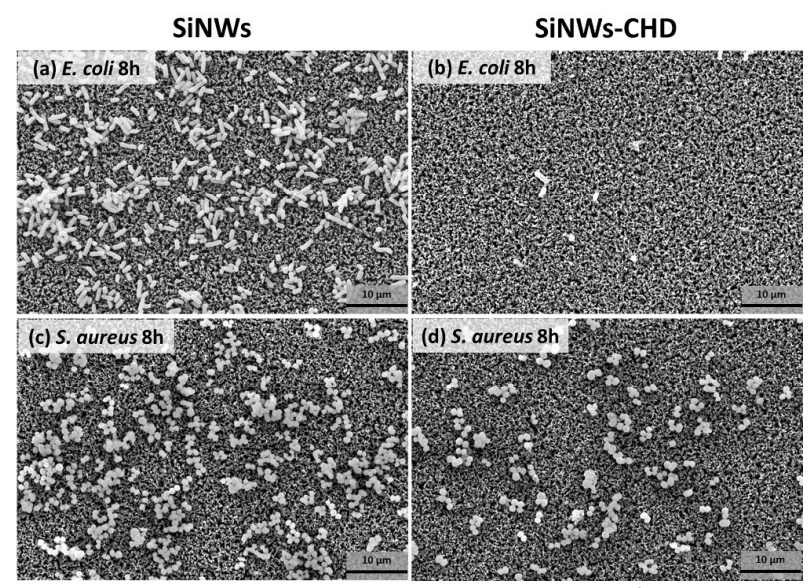

Figure 9. Representative top-view SEM image of $E$. coli and $S$. aureus after 8 h of culture on (a-c) SiNWs and (b-d) SiNWs loaded with $0.02 \% \mathrm{CHD}$.

Comparing viability assays of cells in the planktonic state (Figure 7) and on the surface (Figure 10) we can confirm that in both cases $S$. aureus is less sensitive to CHD, as expected from the higher MIC values obtained in planktonic conditions (Table 1). Samples of SiNW-CHD without APTES functionalization (i.e. SiNWC1 \& SiNWC2) were systematically less efficient against both $S$. aureus and $E$. coli than their SiNWs-APTES (SiNW-A) counterparts. The best antibacterial activity was observed for SiNWs-APTES functionalised with CHD (SiNW-AC1 \& SiNW-AC2). On these SiNWs-APTES surfaces, the biocidal effect of CHD against attached cells was maintained after $24 \mathrm{~h}$, even when the antimicrobial activity against planktonic cells had declined. Interestingly, for all SiNWs-APTESCHD experiments, the viability of re-colonising $S$. aureus cells is strongly compromised, suggesting that a residual activity of CHD at the surface is still present.
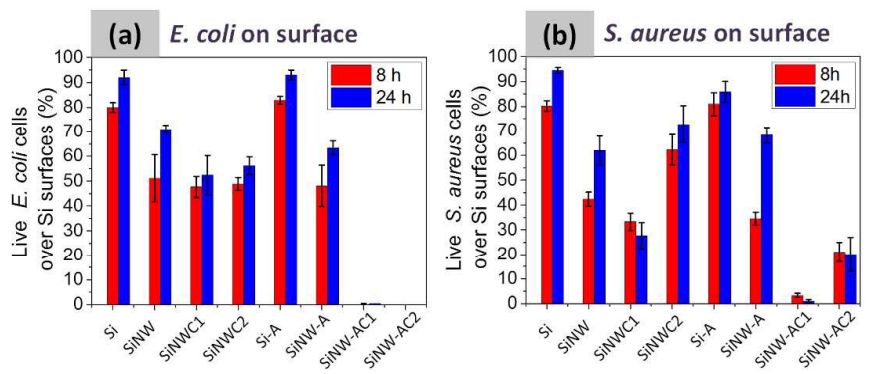

Figure 10. (a) E. coli and (b) S. aureus cell ratios determined with live/dead fluorescent staining. Samples acronyms are listed in Figure 7.

\section{Conclusions}

We have created a three level functionalised surface to study bacterial viability at each level of functionalization on the surface and in planktonic state. Topographical structures in the form of SiNWs and SiNWs-APTES have some intrinsic antibacterial effect, but $E$. coli and $S$. aureus can still proliferate on these topographic surfaces. We have identified two different growth modes producing distinct in plane, and out of the plane bacterial colonies for $E$ coli and S. aureus, respectively. Furthermore, we have incorporated an additional level of functionalization with CHD displaying loadingdependent antibacterial properties. We have demonstrated the role of surface chemistry on the efficacy of this antibacterial effect, with the inclusion of APTES functionalization modifying the release of $\mathrm{CHD}$ and improving antibacterial activity of the SiNWs surfaces.

\section{Acknowledgements}

We would like to thank Dr. Marco Marcello, Dr. Joanna Wnetrzak and Dr. Dave Mason from the Liverpool Centre for Cell Imaging (CCI) for help with experimental design and image analysis support. We also acknowledge the support of University of Liverpool Biomedical Electron Microscopy Unit and Nanoinvestigation Centre at University of Liverpool (NICAL). Gareth Morris from the Open Innovation Hub for Antimicrobial Surfaces and Mattia Morassutto from Mesoscale Chemical Systems (MCS) are acknowledged for assistance in SiNWs-sample preparation. We also thank $\mathrm{S}$. Schlautman (MCS) for sample fabrication and Gerard Kip (NanoLab) 
for XPS-analysis. This work was partly founded by EPSRC grant number EP/J019364/1.

\section{References}

1 N.C. Kyrpides, P. Hugenholtz, J.A. Eisen, T. Woyke, M. Göker, C.T. Parker, et al., Genomic encyclopedia of bacteria and archaea: sequencing a myriad of type strains, PLOS Biol, 2014, 12(8): e1001920

2 M. Wilkins, L. Hall-Stoodley, R. N. Allan, and S. N. Faust, New approaches to the treatment of biofilm-related infections, Journal of Infection, 2014, 69(S1), S47

3 H. Van Acker, P. Van Dijck, and T. Coenye, Molecular mechanisms of antimicrobial tolerance and resistance in bacterial and fungal biofilms, Trends in Microbiology, 2014, 22(6), 326

4 M. Simões, L. C. Simões, M. J. Vieira, A review of current and emergent biofilm control strategies, LWT - Food Science and Technology, 2010, 43(4), 573

5 Biofilms, medical devices, and antibiofilm technology: Key messages from a recent public workshop, American Journal of Infection Control, 2015, 43, 2

6 S. Srey, I. Kabir Jahid, and Sang-Do Ha, Biofilm formation in food industries: A food safety concern, Food Control, 2013, 31, 572e585

7 D. Shchukin, and H. Möhwald, A coat of many functions, Science, 2013, 341.6153, 1458

8 A. Agarwal, T. B. Nelson, P. R. Kierski, M. J. Schurr, C. J. Murphy, C. J. Czuprynski, J. F. McAnulty, and N. L. Abbott, Polymeric multilayer that localize the release of chlorhexidine from biologic wound dressing, Biomaterials, 2012, 33, 6783

9 Z. Zheng, X. Huang, M. Schenderlein, D. Borisova, R. Cao, H. Möhwald, and D. Shchukin, Self-healing and antifouling multifunctional coatings based on $\mathrm{pH}$ and sulfide ion sensitive nanocontainers, Adv. Funct. Mater., 2013, 23, 3307

$10 \mathrm{H}$. Gu, D. Ren, Materials and surface engineering to control bacterial adhesion and biofilm formation: A review of recent advances, Front. Chem. Sci. Eng., 2014, 8(1), 20

11 E. P. Ivanova, J. Hasan, H. K. Webb, G. Gervinskas, S. Juodkazis, V. K. Truong, A. H.F. Wu, R. N. Lamb, V. A. Baulin, G. S. Watson, J. A. Watson, D. E. Mainwaring, and R. J. Crawford, Bactericidal activity of black silicon, Nat. Comm., 2013, 4:2838

12 J. E. Gittens, T. J. Smith, R. Suleiman, R. Akid, Current and emerging environmentally-friendly systems for fouling control in the marine environment, Biotechnology Advances, 2013, 31(8), 1738

13 A. I. Hochbaum, and J. Aizenberg, Bacteria pattern spontaneously on periodic nanostructure arrays, Nano Lett., 2010, 10(9), 3717

14 R. Vasudevan, A. J. Kennedy, M. Merritt, F. H. Crocker, R. H. Baney, Microscale patterned surfaces reduce bacterial fouling-microscopic and theoretical analysis, Colloids and Surfaces B: Biointerfaces, 2014, 117, 225

15 R. J. Crawford, H. K. Webb, V. K. Truong, J. Hasan, E. P. Ivanova, Surface topographical factors influencing bacterial attachment, Advances in Colloid and Interface Science, 2012, $179-182,142$

16 D. B. Weibel, W. R. DiLuzio, and G. M. Whitesides, Microfabrication meets microbiology, Nature Reviews Microbiology, 2007, 5, 209

17 W. Kim , J. K. Ng , M. E. Kunitake, B. R. Conklin, and P. Yang, Interfacing silicon nanowires with mammalian cells, J. Am. Chem. Soc., 2007, 129 (23), 7228

18 Alex K. Shalek, Jacob T. Robinson, Ethan S. Karp, Jin Seok Lee, Dae-Ro Ahn, Myung-Han Yoon, Amy Sutton, Marsela Jorgolli,
Rona S. Gertner, Taranjit S. Gujral, Gavin MacBeath, Eun Gyeong Yang, and Hongkun Park, Vertical silicon nanowires as a universal platform for delivering biomolecules into living cells, PNAS, 2010, 107(5), 1870

19 Minsuk Kwak, Lin Han, Jonathan J. Chen, and Rong Fan, Interfacing Inorganic nanowire arrays and living cells for cellular function analysis, Small, 2015, 11(42), 5600

20 C. Chiappini, E. De Rosa, J. O. Martinez, X. Liu, J. Steele, M. M. Stevens and E. Tasciotti, Biodegradable silicon nanoneedles delivering nucleic acids intracellularly induce localized in vivo neovascularization, Nature Materials, 2015, 14,532

21 C. Liu, J. J. Gallagher, K. K. Sakimoto, E. M. Nichols, C. J. Chang, M. C. Y. Chang, and P. Yang, Nanowire-bacteria hybrids for unassisted solar carbon dioxide fixation to valueadded chemicals, Nano Lett., 2015, 15, 3634

22 N. N. Mishra, W. C. Maki, E. Cameron, R. Nelson, P. Winterrowd, S. K. Rastogi, B. Filanoski, and G. K. Maki, Ultrasensitive detection of bacterial toxin with silicon nanowire transistor, Lab Chip, 2008, 8, 868

$23 \mathrm{Q}$. Yu, H. Liu, and H. Chen, Vertical SiNWAs for biomedical and biotechnology applications, J. Mater. Chem. B, 2014, 2, 7849

24 K. K. Sakimoto, C. Liu, J. Lim, P. Yang, Salt-induced selfassembly of bacteria on nanowire arrays, Nano Lett., 2014, 14 (9), 5471

25 L. Wang, H. Wang, L. Yuan, W. Yang, Z. Wu and H. Chen, Step-wise control of protein adsorption and bacterial attachment on a nanowire array surface: tuning surface wettability by salt concentration, J. Mater. Chem., 2011, 21, 13920

26 Elisabeth Galopin, Gaelle Piret, Sabine Szunerits, Yannick Lequette, Christine Faille and Rabah Boukherroub, Selective adhesion of bacillus cereus spores on heterogeneously wetted silicon nanowires, Langmuir, 2010, 26 (5), 3479

27 K. Q. Peng, H. Fang, J. J. Hu, Y. Wu, J. Zhu, Y. J. Yan, and S. Lee, Metal-particle-induced, highly localized site-specific etching of $\mathrm{Si}$ and formation of single-crystalline Si nanowires in aqueous fluoride solution, Chem.-Eur. J., 2006, 12, 7942

28 S. Schmidt, S. Senz, and U. Gosele, Diameter-dependent growth direction of epitaxial silicon nanowires, Nano Lett., 2005, 5, 931

29 C. Chartier, S. Bastide, and C. Levy-Clement, Metal-assisted chemical etching of silicon in $\mathrm{HF}-\mathrm{H}_{2} \mathrm{O}_{2}$, Electrochimica Acta, 2008, 53, 5509

$30 \mathrm{~K}$. Peng, A. Lu, R. Zhang, and S. T. Lee, Mobility of metal nanoparticles in silicon and induced anisotropic silicon etching, Adv. Funct. Mater., 2008, 18, 3026

31 M. S. A. Rahman, S. C. Mukhopadhyay, P.-L. Yu, J. Goicoechea, I. R. Matias, C. P. Gooneratne, J. Kosel, Detection of bacterial endotoxin in food: New planar interdigital sensors based approach, Journal of Food Engineering, 2013, 114, 346

$32 \mathrm{H}$. Ems, S. Ndao, Microstructure-alone induced transition from hydrophilic to hydrophobic wetting state on silicon, Applied Surface Science, 2015, 339, 137

33 A. Taglietti, Y. A. Diaz Fernandez, E. Amato, L. Cucca, G. Dacarro, P. Grisoli, V. Necchi, P. Pallavicini, L. Pasotti, and M. Patrini, Antibacterial activity of glutathione-Coated silver nanoparticles against Gram positive and Gram negative bacteria, Langmuir, 2012, 28 (21), 8140

34 E. Amato, Y. A. Diaz-Fernandez, A. Taglietti†, P. Pallavicini, L. Pasotti, L. Cucca, C. Milanese, P. Grisoli, C. Dacarro, J. M. Fernandez-Hechavarria, and V. Necchi, Synthesis, characterization and antibacterial activity against Gram positive and Gram negative bacteria of biomimetically coated silver nanoparticles, Langmuir, 2011, 27 (15), 9165 
35 M.-J. Bañuls, R. Puchades, and Á. Maquieira, Chemical surface modifications for the development of silicon-based label-free integrated optical (IO) biosensors: A review, Analytica Chimica Acta, 2013, 777, 1

$36 \mathrm{~F}$. Wu, and Cees Dekker, Nanofabricated structures and microfluidic devices for bacteria: from techniques to biology, Chem. Soc. Rev., 2016, 45,268

37 T. A. Cameron, J. R. Zupan, and P. C. Zambryski, The essential features and modes of bacteria polar growth, Trends in Microbiology, 2015, 23(6), 347

38 P. J. B. Brown, D. T. Kysela, and Y. V. Brun, Polarity and the diversity of growth mechanisms in bacteria, Semin. Cell. Dev. Biol., 2011, 22(8), 790

39 T. J. Silhavy, D. Kahne, and S. Walker, The Bacterial Cell Envelope, Cold. Spring. Harb. Perspect. Biol., 2010, 2(5), a000414

40 K. J. Anusavice, N.-Z. Zhang, and C. Shen, Controlled release of chlorhexidine from UDMA-TEGMA resin, J. Dent. Res., 2006, 85(10), 950

41 H.-Y. Cheung, M. M.-K. Wong, S.-H. Cheung, L. Y. Liang, Y.-W. Lam, S.-K. Chiu, Differential actions of chlorhexidine on the cell wall of Bascillus subtillis and Escherichia coli, 2012, 7(5), e36659 


\section{Graphical Abstract}

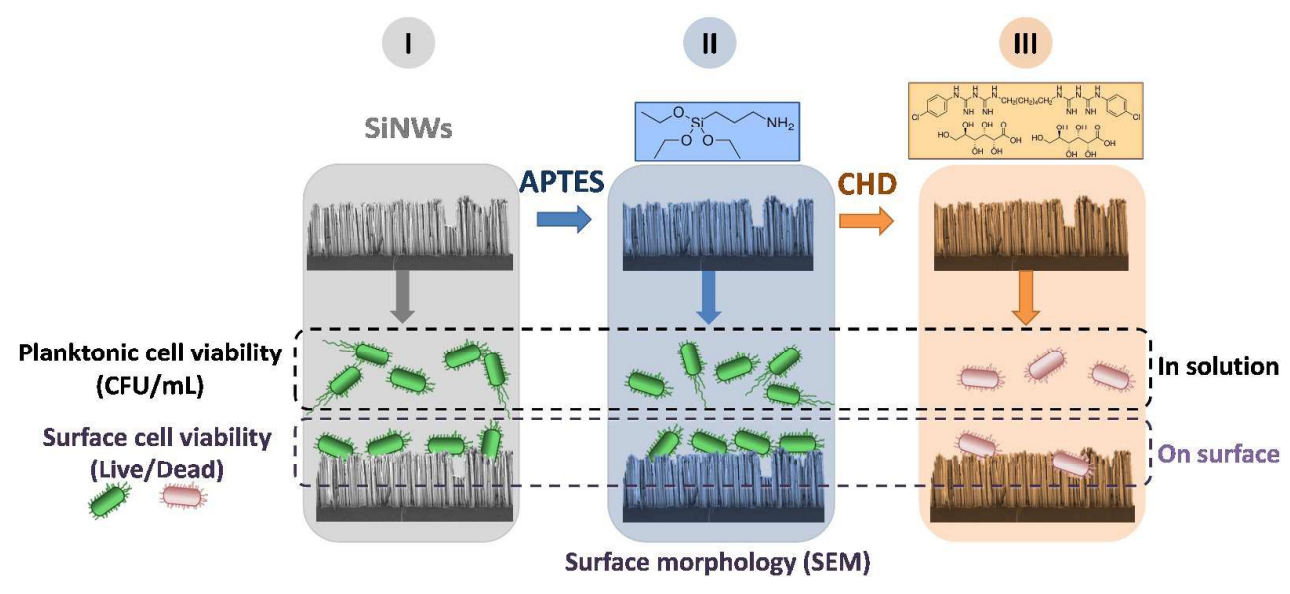

In this study we have fabricated and characterized multiscale-functional nanotopographies with three levels of functionalization: (1) nanostructure topography in the form of silicon nanowires, (2) covalent chemical modification with (3-aminopropyl)triethoxysilane, and (3) incorporation of chlorhexidine digluconate. Cell viability over SiNWs arrays is investigated for E. coli and S. aureus. We have identified two growth modes producing distinctive multicellular structures. We have also shown that chemically modified SiNWs arrays display antibacterial properties. 